\title{
Assessment of the Quality of Care at the Paediatric Dental Clinic of a Tertiary Health Institution in Nigeria
}

\author{
Joycelyn Odegua Eigbobo, ", Felix Emeka Anyiam², Seye Babatunde ${ }^{3}$ \\ ${ }^{1}$ Department of Child Dental Health, Faculty of Dentistry, College of Health Sciences, University of Port Harcourt, Port Harcourt, Nigeria \\ ${ }^{2}$ Centre for Health and Development, University of Port Harcourt, Port Harcourt, Nigeria \\ ${ }^{3}$ Department of Preventive and Social Medicine, Faculty of Clinical Sciences, College of Health Sciences, University of Port Harcourt, Port \\ Harcourt, Nigeria
}

Email address:

odegwabobo@yahoo.com (J. O. Eigbobo), felix.emeka.anyiam@gmail.com (F. E. Anyiam), seyebabs@gmail.com (S. Babatunde)

${ }^{*}$ Corresponding author

\section{To cite this article:}

Joycelyn Odegua Eigbobo, Felix Emeka Anyiam, Seye Babatunde. Assessment of the Quality of Care at the Paediatric Dental Clinic of a Tertiary Health Institution in Nigeria. World Journal of Public Health. Vol. 4, No. 4, 2019, pp. 81-86. doi: 10.11648/j.wjph.20190404.12

Received: May 8, 2019; Accepted: November 15, 2019; Published: November 25, 2019

\begin{abstract}
To evaluate the quality of clinical records as a measure of quality of paediatric oral health care in a tertiary health institution, in Nigeria. A 12-month retrospective review of clinical records of all paediatric dental patients seen in the dental centre of a tertiary hospital in Nigeria was carried out. The clinical records were assessed using a structured standard checklist based on the modified CRABEL Scores. The information assessed in each of the case notes included the case note identification number, patient demographic information, the history of presenting complaint, dental and medical /drug histories. Other information elicited were clinical features, diagnosis, treatment plan, procedures done and professional rank of the person who made the entries. Scores obtained per item for each case note were inputted and analyzed using the Statistical Package for Social Sciences (SPSS) Version 20.0. Three hundred and thirty four clinical records were assessed, of which, 136 (44.3\%), $106(36.8 \%), 65(21.2 \%)$ and $7(2.3 \%)$ of entries were made by students, interns, resident doctors and consultants respectively. The median CRABEL score was $95 \%$. Written consent was the least $(0.3 \%)$ documented information. There were statistically significant differences between the professional rank and extra oral examination, treatment plan and procedures done $(\mathrm{p}<0.05)$. However, there was no significant difference between the professional rank and CRABEL scores $(\mathrm{p}=0.4)$. Using the adequacy of clinical note taking as an index of the quality of care, it may be concluded that the quality of care in our paediatric dental clinic is of high standard. The only shortcoming in our experience was almost the absence of documentation of written informed consent.
\end{abstract}

Keywords: Dental Records, CRABEL Scores, Quality of Dental Care, Nigerian Children

\section{Introduction}

Oral health cannot be separated from general health; it has been described as an essential part of the total health of a child or an adolescent [1]. The quality of oral health care service can be measured in terms of structure (such as facilities, equipment, personnel and administration), process management (diagnosis, treatment planning and other processes that describe how healthcare is delivered), and outcome $[2,3]$. In other words, the quality of care received by individuals could be influenced by the mode of delivery.

In a dental clinic, the quality of care obtainable can be assessed directly through prospective observation by a trained observer or indirectly by retrospective review of clinical records [4]. Although information obtained from the records are useful for medico-legal reasons, forensic purposes [5], planning, and research, their significance in the assessment of quality of care cannot be overlooked $[6,7]$. It has been shown that there is a relationship between the quality of documentation in case files and quality of dental care $[6,8]$. Dental clinical record consists of information pertaining to patient identification, diagnoses made based on comprehensive history, clinical findings, and results of investigations. The treatment plan, treatments carried out, follow-up and home care instructions are also documented 
[9].

Several authors $[10,11]$ have reported inadequacies of dental records; one reason often attributed to this observation is brevity of clinical notes such that vital information on clinical conditions and management were often omitted. This would ultimately affect the quality of care since adequate prior information is required by another practitioner to continue management [6]. Even in situations where the same care provider continues, total recall of previous transactions cannot always be guaranteed $[6,12]$.

Like every standard clinic and hospital, adequate and comprehensive documentation is essential for us at the paediatric dental unit of the University of Port Harcourt Teaching Hospital (UPTH) for continuity of care. This helps to prevent duplication, omissions based on assumptions, and inappropriate treatment [13]. It is particularly important in a tertiary health institution setting like ours where different resident doctors (specialists in-training) and interns rotate through the unit at various times. This study was therefore aimed at evaluating the quality of clinical records as a measure of quality of paediatric oral health care at a tertiary dental centre. Hopefully, the findings will be helpful in formulating appropriate policies on documentation and clinical note taking towards improving the quality oral health care to our paediatric patient population.

\section{Materials and Methods}

\subsection{Study Setting}

The study was carried out at the dental centre of the University of Port Harcourt Teaching Hospital (UPTH), Port Harcourt, Nigeria. The dental centre consists of five clinical departments including the department of Child Dental Health which comprises two units - Paediatric dentistry and Orthodontics units. The paediatric dentistry unit attends to children aged sixteen years and below via a daily clinic system run essentially by resident doctors and interns under the supervision of a specialist paediatric dentist.

\subsection{Study Design/Population and Procedure}

This was a descriptive cross-sectional study conducted over a 12- month period spanning from January to December 2013. A retrospective review of clinical records of all paediatric dental patients seen over the period was carried out. The clinical records were assessed using a structured standard checklist based on the CRABEL Scores (2001) - a set of guidelines for auditing quality of medical records proposed by The Royal College of Surgeons of England and named after the authors (Crawford, Beresford and Lafferty) [14].

The CRABEL scores has been previously modified for dental records audit by Pessian and Beckett [10] and Dosumu et al [15]. In this modified system, an initial score of 100 points was allotted to each case note on the assumption that all necessary information were adequately and appropriately documented. Subsequently, predetermined marks were subtracted from a case note if a required information was found missing. The deductions were done in accordance with the protocol presented in Table 1.

Table 1. Assessment of the quality of Dental records using the modified CRABEL scoring-Crawford, Beresford and Lafferty formulated system.

\begin{tabular}{ll}
\hline Item & Scores subtracted \\
\hline Date & 10 \\
DEMOGRAPHIC DATA & \\
Name, age, gender, marital status, address & 10 \\
Case note number & 10 \\
HISTORY & \\
History of presenting complaint & 5 \\
Medical history & 5 \\
Dental history & 5 \\
Drug history & 5 \\
EXAMINATION & \\
Extra oral & 5 \\
Intraoral & 5 \\
DIAGNOSIS & 5 \\
TREATMENT PLAN & 10 \\
PROCEDURE DONE & 10 \\
VALID CONSENT & 5 \\
SIGNATURE (S) & 5 \\
DEPARTMENT & 5 \\
TOTAL & 100 \\
\hline
\end{tabular}

The information assessed in each of the case notes included the case note identification number, patient demographic information, the history of presenting complaint, dental, and medical /drug histories. Also, clinical features (extra- and intraoral findings), diagnosis, treatment plan and treatments carried out were checked for. The modified system was adopted for this study. The identities of each patient and the clinicians who made the entries were protected even as ethical clearance was obtained from the Research and Ethics committees of the University of Port Harcourt.

\subsection{Data Processing}

Every case note was assessed and scored for each verified item. Scores obtained per items for each case note were inputted and analyzed using the Statistical Package for Social Sciences (SPSS) Version 20.0. Results were presented as frequencies and percentages. Median and Mean scores were computed to determine the overall quality of records at the clinic. ANOVA test was performed to compare the professional rank with information inputted in the clinical notes and CRABEL scores. An observation was considered statistically significant when the probability value is less than or equal to $0.05(\mathrm{p} \leq 0.05)$.

\section{Results}

Three hundred and thirty four clinical records were assessed during the study, of which, 136 (44.3\%), 106 (36.8\%), $65(21.2 \%)$ and 7 (2.3\%) of entries were made by students, interns, resident doctors and consultant, respectively. Twenty $(5.9 \%)$ identities of the operator were not documented. The records fell into different grades of 
quality as determined by the CRABEL scores. Figure 1 illustrates the percentile distribution of CRABEL scores with majority of clinical notes falling within the ninetieth percentile $(90.2 \%)$. Only one-tenth of the records scored below $90 \%$. The minimum and maximum scores were $65 \%$ and $95 \%$, respectively with a mean score of $91.9 \%( \pm 4.8)$ and median score of $95 \%$.

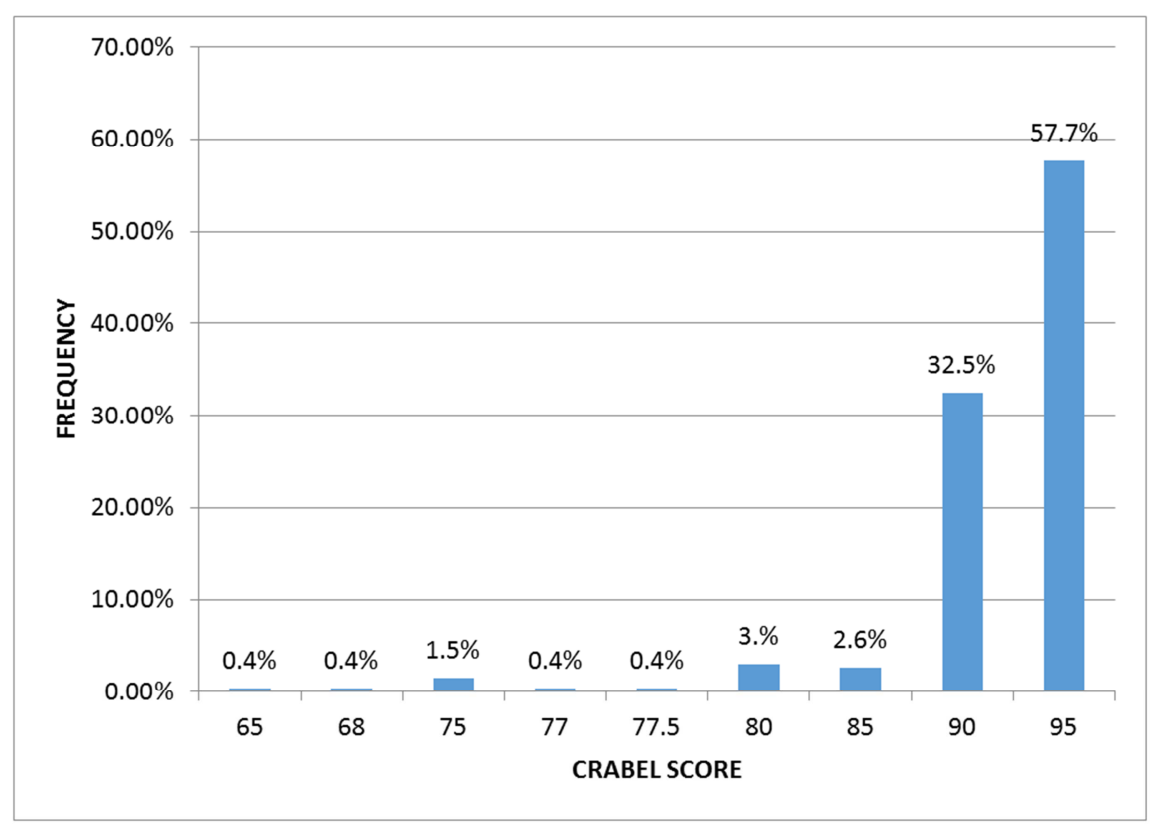

Figure 1. The CRABEL scores of the recordings obtained from the case notes.

When we considered each of the verified items, the date of documentation was correctly entered in 329 (98.5\%) cases while demographic information was recorded in 325 $(97.3 \%)$ cases. Patient identification number was included in all cases. The record of clinical history including history of presenting complaint as well as medical and dental histories were adequately documented in 328 $(98.2 \%)$ cases while drug history was noted in only 210 $(62.9 \%)$. Records of intraoral and extraoral findings on examination were properly documented in $309(92.5 \%)$ and $322(96.4 \%)$ cases, respectively. (Details depicted in Figure 2).

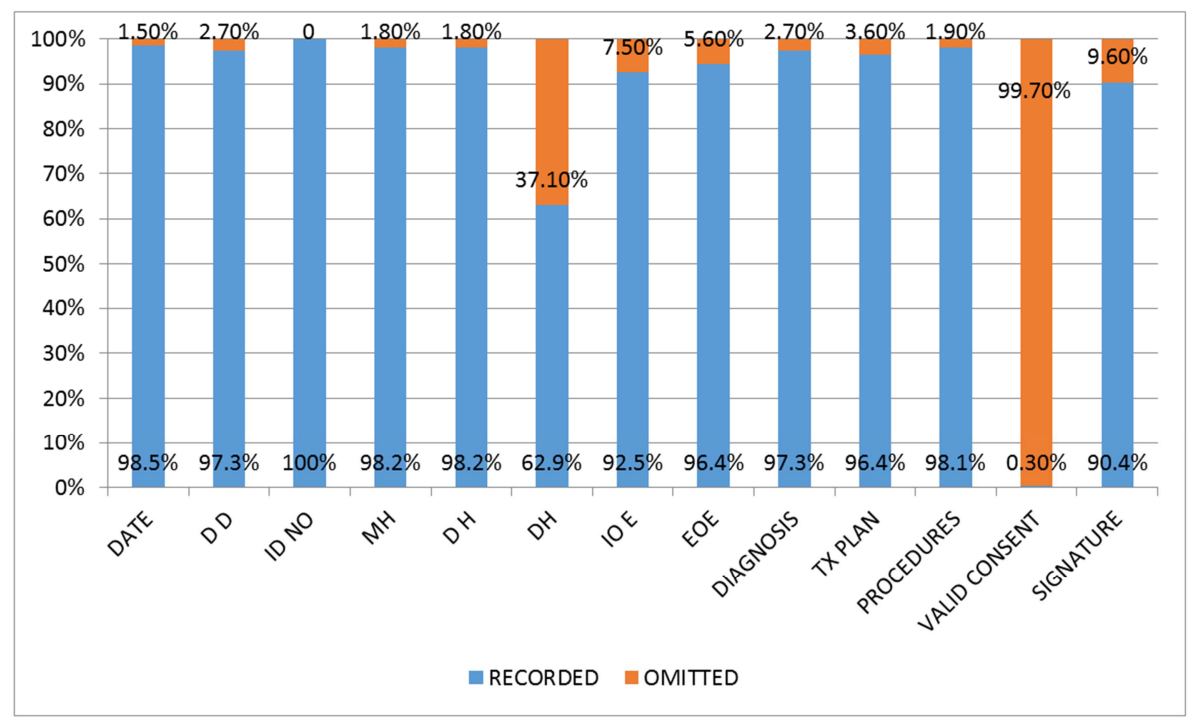

Figure 2. The frequency of recorded and omitted information in the charts of the patients.

DD: Demographic data

EOE: Extra Oral Examination

ID NO: Patient Identification number

TX PLAN: Treatment Plan

MH: Medical History

DH: Dental History

IOE: Intra Oral Examination 
Whereas clinical diagnosis and treatment plans were respectively outlined in $325(97.3 \%)$ and $322(96.4 \%)$ cases, treatment procedures executed were documented in only $261(78.1 \%)$ cases. Interestingly, only one $(0.3 \%)$ record included evidence of a written informed consent obtained from patient prior to treatment. Dentist's name and signature was appended at the end of documentation in $302(90.4 \%)$ cases.

Upon comparing the CRABEL scores of individual notes on the basis of professional ranking using ANOVAtest there was no statistical significant difference $(p=0.40$.) found between ranks, (Depicted in Table 2).

Table 2. The relationship between the professional ranks and the CRABEL scores.

\begin{tabular}{|c|c|c|c|c|c|c|c|c|c|c|}
\hline \multirow{2}{*}{$\begin{array}{l}\text { Professional } \\
\text { rank }\end{array}$} & \multicolumn{10}{|c|}{ CRABEL SCORES } \\
\hline & $65 \mathrm{n}(\%)$ & $75 \mathrm{n}(\%)$ & $77 \mathrm{n}(\%)$ & 77.5 n (\%) & 80 n (\%) & 85 n $(\%)$ & 88 n (\%) & $90 \mathrm{n}(\%)$ & $95 \mathrm{n}(\%)$ & TOTAL n (\%) \\
\hline Students & 0 & $3(2.5)$ & 0 & 0 & $4(3.4)$ & $3(2.5)$ & $1(0.8)$ & $37(31.4)$ & $70(59.3)$ & $118(46.6)$ \\
\hline Interns & 0 & 0 & $1(1.3)$ & 0 & $1(1.3)$ & 2 & $1(1.3)$ & $28(2.6)$ & $43(56.6)$ & $76(30)$ \\
\hline Residents & 0 & 0 & 0 & 1 & 3 & 1 & 1 & 13 & 28 & $47(18.6)$ \\
\hline Consultant & 1 & 1 & 0 & 0 & 0 & 1 & 0 & 4 & $5(41.7)$ & $12(4.7)$ \\
\hline TOTAL & $1(0.4)$ & $4(1.6)$ & $1(0.4)$ & $1(0.4)$ & $8(3.2)$ & $7(2.8)$ & $3(1.2)$ & $82(32.4)$ & $146(57.7)$ & $253(100)$ \\
\hline
\end{tabular}

$* \mathrm{P}=0.40 \mathrm{~F}=1.047$

However, there were statistical significant differences found between ranks when comparing information (Extra oral examination, treatment plan and procedures carried out) in the case notes on the basis of professional ranking (Table 3 ).

Table 3. The relationship between the professional rank and the information elicited from case notes.

\begin{tabular}{|c|c|c|c|c|c|c|}
\hline & Studentsn (\%) & Interns n (\%) & Resident Drs n (\%) & Consultant n (\%) & Total n (\%) & p value \\
\hline Date & $137(99.3)$ & $89(100)$ & $65(100)$ & $13(100)$ & $304 \%(99.7)$ & 0.75 \\
\hline Demography & $138(100)$ & $87(97.8)$ & $64(98.5)$ & $13(100)$ & 302 (99) & 0.61 \\
\hline Id number & $138((100)$ & $89(100)$ & $65(100)$ & $13(100)$ & $303(100)$ & 0.88 \\
\hline Dental H & $135(97.8)$ & $89(100)$ & $64(98.5)$ & $12(92.3)$ & $300(98.4)$ & 0.20 \\
\hline Drug H & $90(65.2)$ & $51(57.3)$ & $43(66.2)$ & $7(53.8)$ & $191(62.6)$ & 0.52 \\
\hline IOE & $136(98.6)$ & 88 (98.9) & $60(92.3)$ & $13(100)$ & $297(97.4)$ & 0.17 \\
\hline Diagnosis & $136(98.6)$ & $89(100)$ & $63(96.9)$ & $13(100)$ & $301(98.7)$ & 0.91 \\
\hline Treatment $\mathrm{P}$ & $137(99.3)$ & $89(100)$ & $61(93.8)$ & $12(92.3)$ & $299(98)$ & $0.03 *$ \\
\hline Procedures & $117(99.2)$ & $76(100)$ & $46(97.6)$ & $11(91.7)$ & $250(98.8)$ & $0.03 *$ \\
\hline Valid consent & $1(0.8)$ & 0 & 0 & 0 & $1(0.3)$ & 0.77 \\
\hline Signature & $126(91.3)$ & $87(97.8)$ & $60(92.3)$ & $12(92.3)$ & $265(93.4)$ & 0.27 \\
\hline
\end{tabular}

$* \mathrm{p}<0.05$-significant

\section{Discussion}

Dental records also known as patient charts are a pool of information on the patient's demographics, disease diagnosis, treatment plan, procedures carried out, referrals and other patient- related communications that occur in the dental office/clinic [6]. They are an essential part of the patient's oral health care relevant both in the present and follow up care of the individual. They are the necessary link between the present and the future, especially where an authorized third party with no prior information about the patient has to continue with the patient management $[9,12]$. In this scenario, a well written clinical note by the previous dentist would help to facilitate easier adaptation and smooth transition between the care providers [16].

In this study, majority of the clinical notes assessed were written by trainees (i.e. resident doctors and interns) who were transiting through their clinical postings at the paediatric dental clinic during the study period. It is therefore necessary that clinical notes are well written for continued care especially as some of the children could still require several visits to the paediatric clinic before growing into adulthood when they might transit to adult dental clinics. Even adolescents at the terminal visits to paediatric clinic, require proper records for effective transfer to adult dental care.

A review of the evidence from studies carried out in the USA, Australia and Scandinavia showed that record keeping often fell below accepted standards [8]. Hence, there was the need to assess clinical records in an objective, effective and reproducible manner. This led to the development of a standardised scoring system by Crawford, Beresford and Lafferty (CRABEL) which has been adopted by the Royal College of Surgeons. This criteria scoring system which was initially exclusive for medical record auditing has been modified and used for auditing dental records.

This study showed that the quality of clinical note-taking in our clinic based on the CRABEL scoring system is very good. With over $90 \%$ of clinical notes scoring within the $90^{\text {th }}$ percentile, suggestively, only minimal remediation is required to optimize the note-taking capacities of working personnel at the clinic. This observation is similar to an earlier report from the University College Hospital Ibadan in 
Nigeria [15] but sufficiently different from reported scores with students in a UK study [10]. The similarity of observations from the two Nigerian institutions could be partly explained by the uniformity of training curriculum in the country where the norm is supervision by superior officers.

Indication of department and identification numbers in case notes is statutorily imposed at the point of registration in our settings. This certainly accounts for $100 \%$ compliance in our study as well as that reported among undergraduate students in Ibadan, Nigeria [15]. Again, this observation contrasts the experience of Pessian and Beckert [10] who reported that notation of departments was most commonly omitted by dental undergraduates in their own study.

It might sound alarming to note that record of a valid (written) consent was documented in only one case note in the present study. A similar observation was made in the study conducted at Ibadan [15] in which case not even a single documentation of written consent was found. This observation is easily explained by the fact that obtainment of written consent for non invasive procedures is not yet a routine in our practice in Nigeria. It is often said that most routine procedures carried out in the paediatric dental clinics are non invasive and so may not require written consent, unless the use of conscious sedation and/or general anaesthesia is involved [17]. Incidentally, cases requiring this mode of analgesia/anaesthesia are often surgical and reasonably invasive, hence there would be good reasons for insisting on written consent [18]. The only case of documented consent in our study was actually for an invasive surgical procedure. However, since paediatric dental patients are generally minors, besides the implied consent facilitated by consultation and submission of children by their parents for examination and treatment by dentists, verbal consent is often always sought and received from parents or designated adults.

In an earlier publication, the extent of implied consent in dental practice was defined as; an individual entering a dental surgery, sitting and allowing dental examination only [19]. Nevertheless, whether consent is implied, verbal or expressed in writing, it is always important to ensure informed consent is obtained after due explanations of procedures, possible complications and outcomes [20, 21]. This is so important considering that verbal consent is not admissible in a court of law and can actually be denied by the parents irrespective of any cultural or moral reasons for justifying it.

It was also observed that the names or signatures of the individuals involved were omitted in about a tenth of the case notes. The study carried out by Dosumu et al [15] had signatures in all the case notes this may be because the students had to be supervised and the supervisor had to sign. In this present study the entries were not done exclusively by students. One may therefore deduce that the omitted signatures were by qualified dentists (residents and consultant) since a student's entry had to be counter signed.

\section{Conclusion}

Using the adequacy of clinical note taking as an index of the quality of care, it may be concluded that the quality of care in our paediatric dental clinic is of high standard as justified by the high CRABEL scores of our clinical notes. The only shortcoming in our experience was the absence of documentation of informed consent. This has been explained by the lack of a mandating policy for such documentation.

\section{Recommendations}

A review on the aspect of obtaining informed consent is needed to ensure adherence to the best practice of obtaining expressed consent for every procedure, invasive or otherwise. However, the concept of quality of care is multivariate, while clinical note taking may be a significant variable, we remain conscious of all other factors that may impact on the overall quality of care in a clinical setting.

\section{Conflict of Interest Statement}

None of the authors have any conflicts of interest that should be disclosed.

\section{References}

[1] U. S. Department of Health and Human services. Oral health in America: a report of the surgeon general. Rockville: U. S. Department of Health and Human Services, National Institute of Dental and Craniofacial Research, National Institutes Of Health; 2000.

[2] A. Adindu and S. Babatunde (2006). Health Managers Perception of the Primary Health Care Management Information System: A case of Bama Local Government in Northern, Nigeria. Nigerian Journal of Medicine15: 266-271.

[3] A. Donabedian (1988). The quality of care: How can it be assessed? Journal of the American Dental Association, 11, $1145-1150$.

[4] Indian Health Services. Oral Health program guide. Quality Assessment and improvement. Appendix III Chapter 7: Indirect review of Clinical Quality Chart Review. 2007: 1-15.

[5] B. K. Charangowda (2010). Dental records: an overview. Journal of Forensic Dental Sciences 2, 5-10.

[6] New York University. Chapter 10: Dental Records. http://www.nyu.edu/dental/careers/.../startingyourdentalpractic e/chapter10.pdf. Pp 79-86. Accessed 02/09/16.

[7] J. Crall (1989). Evaluations of effectiveness and quality of care. Journal of Dental Education53, 673-676.

[8] R. G. Morgan (2001). Quality assurance: Quality evaluation of clinical records of a group of general dental practitioners entering a quality assurance programm. British Dental Journal191, 436-441.

[9] American Academy of Pediatric Dentistry (2009). Guideline on record keeping. Pediatric Dentistry 2008-2009; 30: 226233. 
[10] F. Pessian andH. A. Beckett (2004). Record keeping by undergraduate dental students: A clinical audit. British Dental Journal197, 703-705.

[11] S. E. Heilminen, M. Vehkalahti, H. Murtomaa, P. Kekki and T. M. Ketomäki (1998). Quality evaluation of oral health recordkeeping for Finnish young adults. Acta Odontologica Scandinavica, 56, 288-292.

[12] A. Dierickx, M. Seyler, Valck E. De Wijffels J and G. Willems (2006). Dental Records: A Belgium Study, Journal of Forensic Odontostomatology 24, 22-31.

[13] A. Narayanan, N. Ashok and K. Sebastian (2011). A study of patient record documentation, practice of dentists in Kerala. Kerala Dental Journal34: 430-432.

[14] J. R. Crawford, T. P. Beresford andK. L. Lafferty (2001). The CRABEL score - a method for auditing medical records Annals of Royal College of Surgeons of England 83, 65-68

[15] E. B. Dosumu, O. O. Dosumu and F. B. Lawal (2012). Quality of records keeping by undergraduate dental students in Ibadan, Nigeria, Annals of Ibadan Postgraduate Medicine10, 13-17.

[16] World Health Organization- Western Pacific Region. Medical
Records Manual/A Guide for Developing Countries. Accessed 23/01/16

http://www.wpro.who.int/publications/docs/MedicalRecordsM anual.pdf.

[17] Australian Dental Association Inc. Guidelines for good practice on consent for care in dentistry. Accessed 23/01/16 http://www.ada.org.au/app_cmslib/media/lib/0703/m52394_v 1_ggp2consentforcareindentistry.pdf.

[18] J. T. Arotiba, V. I. Akinmoladun and V. N. Okoje (2006). An audit of medical record-keeping in maxillofacial surgery at the University College Hospital, Ibadan, using the CRABEL scoring system. African Journal of Medicineand Medical Sciences 35, 93-95.

[19] P. Lambden (2002). Dental law and ethics. Radcliffe Medical press 1td. Abingdon. UK. ISBN 1-85775-911-7. Pp 81-83.

[20] American Dental Association Division of Legal Affairs. Dental Records. Chicago, Ill. American Dental Association; 2007: 16.

[21] P. Sfikis (2003). A duty to disclose: Issues to consider in securing informed consent. Journal of American Dental Association 13, 1329-1333. 